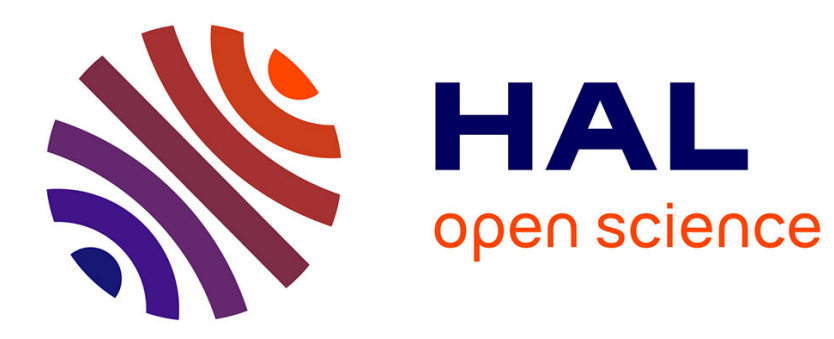

\title{
L'enseignement en FLE de la phraséologie du lexique des affects
}

Cristelle Cavalla, Virginie Labre

\section{To cite this version:}

Cristelle Cavalla, Virginie Labre. L'enseignement en FLE de la phraséologie du lexique des affects. Novakova I. \& Tutin A. Lexique des émotions, Ellug, pp.297-316, 2009. hal-00699919

\section{HAL Id: hal-00699919 https://hal.science/hal-00699919}

Submitted on 8 Feb 2019

HAL is a multi-disciplinary open access archive for the deposit and dissemination of scientific research documents, whether they are published or not. The documents may come from teaching and research institutions in France or abroad, or from public or private research centers.
L'archive ouverte pluridisciplinaire HAL, est destinée au dépôt et à la diffusion de documents scientifiques de niveau recherche, publiés ou non, émanant des établissements d'enseignement et de recherche français ou étrangers, des laboratoires publics ou privés. 


\title{
L'enseignement en FLE de la phraséologie du lexique des affects
}

\author{
Cristelle Cavalla, Virginie Labre
}

Lidilem - Université Stendhal-Grenoble3

\section{Résumé}

Ce travail aborde la question de l'enseignement/apprentissage des expressions associées aux affects à des apprenants non francophones. Mémoriser puis utiliser cette phraséologie demande à l'apprenant un travail préliminaire de reconnaissance de la forme morphosyntaxique de ces structures particulières, puis un travail de décodage et de reconnaissance du sens. Nous aborderons quelques aspects de la description linguistique des phénomènes phraséologiques pour aboutir à des réflexions didactiques pour leur enseignement en Français Langue Etrangère.

\begin{abstract}
This work tackles the question of teaching/training of the expressions associated with the affects to non-native speaker learners. Memorizing and using this phraseology requires a preliminary work of morphosyntactic pattern recognition from these particular structures, then a task of decoding and recognition of the lexical meaning. We will approach some aspects of the linguistic description of the phraseological phenomena which will lead to didactic proposals for teaching them in courses of French as Second Language.
\end{abstract}

\section{Introduction}

L'enseignement/apprentissage, à des apprenants non francophones, des expressions associées aux affects, fait appel à plusieurs aspects linguistiques pour leur description - notamment la sémantique et la syntaxe pour leurs aspects combinatoires - ainsi qu'à plusieurs points de vue didactiques pour leur enseignement notamment en Français Langue Etrangère (désormais FLE). La reconnaissance de la forme morphosyntaxique de ces structures phraséologiques nécessite pour l'enseignant de FLE de proposer des descriptions linguistiques du phénomène, descriptions sur la langue française qui intéressent désormais nombre de linguistes. En outre, l'apprenant aura à décoder le sens de ces expressions souvent imagées et peu motivées sémantiquement (même si les collocations sont davantage motivées sémantiquement que les expressions figées). L'enseignant de FLE a peu de moyens à sa disposition, contrairement à l'enseignant d'anglais langue étrangère qui bénéficie de plusieurs ouvrages didactiques qui traitent du sujet. Sans avoir la prétention de donner des solutions "clé en main » aux enseignants de FLE, nous aborderons quelques aspects de la description linguistique des 
phénomènes phraséologiques autour des affects, pour aboutir à des réflexions didactiques pour leur enseignement en FLE.

Notre réflexion et notre problématique se situent au carrefour de deux disciplines : la linguistique et la didactique. Nous tenterons de répondre aux questions suivantes :

Au plan linguistique : quel type de structures nous intéresse et qu'est-il pertinent de mettre en avant pour aider à la mémorisation et à la bonne utilisation de ces structures?

$\mathrm{Au}$ plan didactique : comment enseigner la phraséologie liée aux affects à des étudiants non francophones?

Notre première interrogation touche à l'aspect linguistique du phénomène et conduit à chercher des traits linguistiques saillants spécifiques à cette phraséologie des affects. Un tel savoir linguistique permettrait davantage de clarté méthodologique pour l'enseignement du FLE (classement spécifique, particularités à noter pour certaines expressions...). Les travaux de Mel'čuk et al. (1996), Gonzalez-Rey (2002), Tutin et al. (2002), Grossmann et al. (2003), Cavalla et al. (2005), Siepman (2006) nous ont aidées à mieux comprendre et cerner certains de ces phénomènes linguistiques.

$\mathrm{Au}$ plan didactique, les réflexions et les constats d'enseignants étrangers de français ont permis de mettre en évidence certaines difficultés d'apprentissage face à ces expressions. Des enseignants de FLE, locuteurs non-natifs (LNN), avouent avoir du mal à retenir ces structures car ils ne savent pas toujours à quelle notion les associer exactement et préfèrent, dans ce cas, ne pas les utiliser. Cependant, ils savent que leur emploi est souvent un raccourci linguistique pour exprimer quelque chose qui prendrait davantage de mots voire de phrases si l'expression n'existait pas. Ils sont donc friands de ces expressions et voudraient pouvoir les enseigner de façon plus efficace à leurs étudiants étrangers apprenants de français. Plusieurs chercheurs se sont penchés sur l'enseignement de ces structures et, dans l'espoir de s'approcher d'un apprentissage raisonné, nous proposerons une brève présentation de quelques-uns de leurs travaux : ceux de Galisson (1984), Lewis (2000), Binon et al. (2003), Verlinde et al. (2006). À partir de ces exemples, nous avons élaboré des séquences didactiques pour l'enseignement du lexique des affects de façon générale (Cavalla et al., 2005) et de la phraséologie des affects en particulier (Labre, 2006).

Dans un premier temps, nous présenterons les descriptions linguistiques qui nous intéressent pour l'enseignement en FLE. Dans un deuxième temps, nous regarderons les travaux de chercheurs à ce propos et enfin, dans un troisième temps, nous discuterons des propositions que nous avons faites pour l'enseignement/apprentissage de la phraséologie des affects.

\section{Descriptions linguistiques}

Plusieurs typologies des éléments de la phraséologie ont vu le jour comme celle de GonzalezRey (2002) qui propose un classement des différentes structures (collocations, expressions idiomatiques, parémies...) ; celle de Mel'čuk qui distingue les "phrasèmes complets » sans compositionnalité des «semi-phrasèmes » pour les collocations aux « quasi-phrasèmes » où le sens du tout exprime davantage que le sens des parties (Tutin, et al., 2002); celle de Cowie (1978) où il décrit des «open collocations » (quasiment toutes les combinaisons de lexies au sens large du terme) et des «restricted collocations» (les combinaisons de lexies dans lesquelles le sens de l'une limite les possibilités d'apparition de l'autre); enfin, le travail de 
Tutin et al. (2006) qui s'attache aux noms de sentiments et à leur combinatoire collocative, qui n'est pas une typologie de la phraséologie mais qui se sert - entre autre - de la phraséologie pour classer les noms de sentiments, ce qui est intéressant au plan didactique.

Pour l'enseignement, nous préciserons le type de structures phraséologiques qui nous intéressent et tenterons d'expliquer en quoi la description linguistique de ces éléments vient éclairer l'enseignement.

Toutes les formes de phraséologie décrites par les chercheurs apparaissent logiquement dans la description des affects. Nous nous intéresserons essentiellement aux expressions figées ( $l a$ moutarde qui monte au nez, nid d'ange) et aux collocations (être aux anges, procurer du bonheur) qui sont deux types de structures. Il existe plusieurs définitions de ces éléments linguistiques, pour les collocations nous nous arrêterons sur celle de Tutin et al. (2002) qui rassemble plusieurs réflexions à ce propos. En outre, ces définitions sont intéressantes au plan didactique, ce que nous verrons plus tard.

\subsection{Combinatoire syntaxique}

Les différents chercheurs cités précédemment ont décrit certaines des formes syntaxiques de ces éléments phraséologiques et il en ressort plusieurs points qui intéressent notre objectif didactique. Tant l'expression figée que la collocation sont des associations lexicales de plusieurs mots graphiques (EF : cordon bleu, les cheveux qui se dressent sur la tête; collocations : prêter attention, prendre peur). Cependant, des caractéristiques syntaxiques séparent les deux phénomènes comme suit.

L'expression figée ne souffre quasiment aucun élément externe entre ses composants (notons qu'il est souvent possible pour les verbes d'introduie un adverbe : Il n'a pas pris ses jambes à son cou) ; quand cela ce produit il s'agit alors de figement très contraint sémantiquement au sein de l'expression (un cordon très bleu: le sens redevient propre). Cette cooccurrence d'éléments n'est pas prédictible, ce qui rend les expressions figées difficiles à produire pour des LNN : il est impossible de prévoir quels mots associer les uns aux autres pour produire le sens recherché d'autant plus que la combinatoire syntaxique de ces éléments peut être très complexe : Prendre ses jambes à son cou $(\mathrm{V}+$ dét. $+\mathrm{N}+$ prép. + dét. $+\mathrm{N})$.

La collocation se définit davantage comme une " cooccurrence lexicale privilégiée de deux éléments linguistiques entretenant une relation syntaxique [...] ferme intention, brouillard à couper au couteau, prêter attention ». (Tutin, et al., $2002: 2$ ). Une telle association lexicale est dite «prédictible » dans sa forme dès l'instant où tout LN va - généralement - deviner la fin de la collocation (ce qui n'est pas le cas d'un LNN). Enfin, trois caractéristiques des collocations nous paraissent majeures pour l'enseignement :

1. les collocations acceptent (le plus souvent) l'introduction d'éléments extérieurs en leur sein : entraîner des conséquences ou entraîner de nombreuses conséquences, le sens de la collocation n'est pas profondément affecté ;

2. leur combinatoire syntaxique peut être prédictible dans certains cas : les noms d'affects ont des collocations associées dont la combinatoire reste fixe dans un paradigme lexical limité (ce qui facilite l'accès pour l'enseignement et l'apprentissage); par exemple Vdans + dét. + N_Affect (collocation verbale) : Nager dans le bonheur ou Nde + N_Affect (collocation nominale) : Un élan de bonheur (Tutin, et al., 2006) 
3. leur structure morphosyntaxique peut varier ou être fréquemment inattendue. Tutin a montré que «la collocation fonder une analyse sur ${ }^{l}$ se rencontre autant dans sa construction canonique (voix active) que dans des constructions passives (l'analyse est fondée sur) ou moyennes (l'analyse se fonde sur)»(Tutin, 2007 : 247). De telles régularités peuvent être exploitées dans l'enseignement du FLE.

\subsection{Compositionnalité sémantique}

L'un des points divergents entre expression figée et collocation est la compositionnalité sémantique. Rapidement, lors de la production et de l'analyse, apparaît un clivage entre la réception sémantique des expressions figées et des collocations, ce qui entraîne une production différente de ces éléments par des LNN. Au plan didactique, on s'aperçoit que plus la phraséologie a un sens prédictible et donc accessible (car peu métaphorique ou peu culturellement marqué) en réception, et plus la production (le réemploi) sera aisée. De ce point de vue, la majorité des collocations paraissent plus faciles à comprendre que les expressions figées.

Pour les expressions figées, le sens de l'ensemble n'est pas compositionnel et très difficilement prédictible; la forme aussi n'est pas prédictible. Ainsi, est-il impossible de prévoir bleu après (ou d'après) cordon pour obtenir le sens recherché dans cordon bleu. En outre, tous les éléments de l'expression ont soit une valeur métaphorique soit une valeur culturelle fortement marquée (références historiques ou littéraires ou mythologiques non partagées par le monde entier) ce qui en complique l'accessibilité sémantique.

Pour les collocations en revanche, le sens de l'association reste parfois prédictible ce qui facilite l'accès sémantique aux LNN. Cette propriété est liée au fait que l'un des éléments de la collocation conserve son sens habituel (la base) tandis que l'autre (le collocatif) prend une valeur plus ou moins opaque (dans entraîner une conséquence, conséquence conserve son sens propre tandis que entraîner est métaphorisé). De ce fait, le sens propre d'un élément aide à la compréhension - même partielle dans un premier temps - de l'ensemble. Selon Hausmann (Hausmann, 1989) les collocations sont quasiment « transparentes »; ceci est peutêtre vrai pour des LN, cependant, l'expérience dans l'enseignement du FLE nous porte à pencher pour la réserve de Mel'čuk qui estime que certaines sont au contraire complexes telle que peur bleue. Verlinde également considère que la motivation sémantique, souvent absente, rend "difficilement compréhensibles et prédictibles [ces structures] pour l'apprenant» (Verlinde et al., 2006). Ajoutons que pour un LNN feuilleter un livre n'est évident au plan sémantique que si le contexte l'aide à extraire le sens, mais la collocation isolée parait toujours « bizarre » aux LNN.

Cette question de la compositionnalité sémantique a conduit à une enquête (Labre, 2006) auprès de LN et de LNN afin de décrire le rapport entre la compréhension de la phraséologie des affects et le degré d'opacité sémantique de ces expressions : plus le degré métaphorique est élevé et plus l'expression a un sens difficile d'accès pour des LNN. Il en résulte que plus l'opacité est grande et moins les LNN ont accès au sens hors contexte. Cependant, ce constat ne préjuge pas de l'appropriation de ces expressions selon leur degré d'opacité. Il semble que pour les LNN, opacité soit synonyme d'une capacité limitée à traiter le sens des éléments. Elle est aussi synonyme de perplexité face à l'expression/collocation dont le sens reste un mystère

\footnotetext{
${ }^{1}$ Test effectué sur un corpus d'articles d'1 $\mathrm{M}$ de mots.
} 
malgré - parfois - la connaissance du sens propre des éléments en présence. Nous pouvons donc dire que l'opacité semble, pour les LNN, un élément bloquant pour déduire du sens. Ceci vient compléter l'affirmation de Verlinde et Binon au plan sémantique : «Le sens de la combinaison est-il égal ou non à la somme des composants. La décision à prendre est, dans un certain nombre de cas, partiellement subjective. » (Verlinde, et al., 2006 : 92). En outre, l'enquête révèle que les $\mathrm{LN}$ n'hésitent pas à employer des expressions figées et des collocations dont ils ne maîtrisent pas le sens. En revanche, un LNN n'osera pas prendre le risque d'employer une expression qu'il maîtrise mal, que tard dans son apprentissage. La performance des LNN est victime de l'insécurité linguistique à exprimer des éléments mal maîtrisés tant au plan syntaxique que sémantique.

\subsection{Vers l'enseignement}

L'enquête de Labre a révélé que la dichotomie compétence/performance n'est pas forcément liée à un niveau haut d'opacité sémantique. On observe que s'il existe un lien étroit entre opacité élevée et lacunes dans la compétence et la performance comme pour Avoir les foies, ce phénomène n'est pas une généralité. En effet, une expression de faible opacité sémantique, ne conduit pas systématiquement à son utilisation. Tel est le cas pour Chercher des poux dans la tête de quelqu'un où les LNN déclarent comprendre l'expression mais ne pas l'utiliser (ni même la connaître pour une grande majorité). D'un point de vue didactique, cet élément est important à noter puisqu'il indique que les expressions figées et les collocations classées «faciles» (par les LNN) car peu opaques posent malgré tout des soucis d'apprentissage et d'emploi aux LNN.

Ces caractéristiques linguistiques permettent de dégager deux grandes tendances pour l'enseignement de ces éléments :

Pour l'enseignement des collocations, il semble important de tenir compte de la combinatoire syntaxique mais au sein d'un champ sémantique particulier (les affects par exemple). Il faut user de la prédictibilité sémantique des éléments dès que le sens propre de la base aide significativement à déduire le sens du collocatif. Cependant, il semble falloir conserver à l'esprit que l'accès au sens n'est jamais simple et que la motivation sémantique présente dans les collocations n'est pas toujours aussi évidente qu'on pourrait le croire pour les LNN. Ce dernier point demandera certainement une réflexion didactique à propos des représentations contenues dans cette phraséologie.

Pour l'enseignement des expressions figées, les spécificités linguistiques étant moins prédictibles, il sera alors nécessaire d'envisager une mise en discours beaucoup plus systématique afin que le contexte aide au mieux à extraire le sens de ces éléments.

L'enseignant de langue étrangère doit alors pouvoir guider ses apprenants vers $1 /$ le repérage de ces éléments dans les discours, 2/ la présentation de leurs structures syntaxiques et lexicales, 3/ l'explication de leur sens, 4/ leurs particularités discursives afin de mieux comprendre pourquoi tel énoncé et telle situation de communication contient plutôt telle collocation ou expression figée que telle autre comme le précise Binon :

«savoir comment on communique dans telle ou telle situation de communication: vocabulaire, constructions syntaxiques, formules de communication, aspects stratégiques, conventions en vigueur, etc. » (Binon, $2000: 27$ ) 
Ainsi l'enseignement va-t-il privilégier les structures phraséologiques qui entrent dans des discours variés tant par leur sujet que par l'utilisation de ces structures (on ne dit pas sa joie de la même façon selon le sujet : une réussite professionnelle, un anniversaire...). Par ce biais, l'enseignant tentera de conduire ses apprenants vers la maitrise du discours de différentes situations de communication.

\section{L'enseignement/apprentissage de la phraséologie des affects}

\subsection{Constat : un enseignement limité}

Expressions figées et collocations sont aussi courantes l'une que l'autre dans le langage quotidien (il existe davantage de collocations que d'expressions figées, mais elles ont autant de chance d'être utilisées). Néanmoins, les collocations et les expressions figées sont peu prises en compte dans l'enseignement du FLE malgré leur importance dans la langue comme le constate Lewis pour les collocations en anglais et pour l'ensemble des langues étrangères :

« $\mathrm{We}^{2}$ now recognise that much of our 'vocabulary' consists of prefabricated chunks of different kinds. The single most important kind of chunk is collocation. Self-evidently, then, teaching collocation should be a top priority in every language course. » (Lewis, 2000)

Afin de développer cet enseignement, il serait intéressant de faire prendre conscience, tant aux enseignants qu'aux apprenants, de l'importance de ces structures dans la langue. Comme le mentionne González-Rey (2002), en langue maternelle ces structures spécifiques sont «des 'béquilles' qui sédimentent automatiquement notre fonds linguistique et dont nous nous servons commodément, sans effort, ce qui semble [leur] ôter toute importance »; en revanche, " elles représentent de véritables entraves dans l'acquisition de la nouvelle langue, contre lesquelles bute sans cesse notre esprit en quête de logique, ce qui leur confère une valeur particulière ». Malgré ce constat déjà évoqué par Bally en 1909 (González-Rey, 2002), l'apparition tardive, et souvent anecdotique, de ces structures dans les méthodes d'enseignement (niveaux avancés en langue $\mathrm{B} 2$ à $^{3}$ ), prouve le peu d'importance qui leur est accordé dans l'apprentissage alors qu'elles font partie intégrante du lexique. Ce phénomène linguistique fait appel à des notions très intéressantes et importantes à prendre en compte dans l'enseignement d'une langue-culture d'autant plus qu'il touche aux représentations conjointes des LN (et de l'enseignant natif) et des LNN.

Certaines études sur l'acquisition précoce des langues maternelles décrivent les capacités de très jeunes LN (d'environ 2 ans) à comprendre trois fois plus de lexique que ce qu'ils en produisent (Bassano, 2005). En FLE, les enseignants le constatent rapidement dans leurs cours: les apprenants comprennent rapidement les consignes, les documents, mais ne franchissent que plus tardivement le pas vers la production (surtout orale) de ces acquis (notons que ce constat est apparemment valable pour l'enseignement des langues étrangères de façon plus générale selon Gass et al. (2001)). Ceci est d'autant plus difficile pour

2 « Nous savons désormais qu'une grande part de notre 'vocabulaire' est constituée de différentes structures préfabriquées. Les plus simples d'entre elles sont les collocations. En conséquence, nous pensons que l'enseignement des collocations devrait être une priorité dans tous les cours de langues étrangères. » (Traduction personnelle)

3 Niveaux décrits dans le CECRL: Cadre Européen Commun de Référence en Langue, www.coe.int/t/dg4/linguistic/Source/Framework_FR.pdf 
l'acquisition des unités phraséologiques ne serait-ce que par leur taille (plusieurs lexies à retenir simultanément). De ce fait, l'apprentissage chez l'apprenant doit être envisagé non seulement grâce à la mémorisation de lexies, mais aussi grâce à la manipulation de ces structures particulières de façon plus développée encore que pour les unités lexicales isolées. Ceci aiderait l'apprenant de langue étrangère à développer une compétence lexicale qui l'entraînerait à davantage reconnaître les structures plus figées que d'autres.

\subsection{La place de la phraséologie des affects dans les discours}

Le LN a tendance à attribuer un caractère oral aux expressions figées. C'est peut-être en raison de cette représentation langagière que ces structures n'apparaissent que très peu dans les méthodes d'enseignement du FLE. Certaines études (Bidaud, 2006) ont cependant montré que ces associations lexicales sont fréquentes dans les écrits journalistiques. Les allusions culturelles faites par les journalistes dans les titres de leurs articles, voire dans la totalité de leurs articles, reposent parfois sur une expression ou une collocation souvent impossible à décoder par un LNN non averti. González-Rey constate que ces "croyances populaires » placent ces expressions dans les registres courant, familier, voire vulgaire et argotique, ce qui reste une croyance car ces structures apparaissent dans tous les registres de langue. Elle mentionne l'exemple de Rire très fort: «1/ «Soutenu : Rire à gorge déployée ; 2/ Courant: Rire aux larmes ; 3/ Familier : Rire comme un bossu ; 4/ Vulgaire : Se fendre la poire ; 5/ Argotique : Se troncher la gueule ${ }^{4}$. Il faut donc renoncer à considérer le registre familier ou populaire comme une caractéristique propre aux expressions figées. » (González-Rey, 2002).

Notons que ces considérations affectent davantage les expressions figées que les collocations. Les collocations souffrent de leur fréquence et les LN ne se rendent pas compte de l'utilisation de telles structures (aspect évoqué précédemment sur la prise de conscience nécessaire de l'existence de ces structures). Les auteurs de manuels d'enseignement du FLE sont dans ce cas et n'ont pas encore tous les moyens linguistiques pour envisager l'enseignement des collocations ; mais comment enseigner un élément dont on n'envisage pas l'existence?

Pour l'enseignement, il serait donc nécessaire de mettre en exergue les collocations dans un premier temps. Celles qui sont associées aux affects sont d'autant plus intéressantes qu'elles apparaissent dans des situations de communications très variées en raison de la notion d'affect elle-même. En effet, on peut trouver des affects dans quasiment toutes les situations de communication sociales et divers types de collocations vont alors apparaître aux côtés d'expressions figées propres elles aussi à la situation. Par exemple, enseigner le lexique de la joie demande que l'on s'arrête sur une des situations suivantes : la joie de réussir un examen, celle de fêter un anniversaire ou encore la joie de vivre. Dans ces trois situations, nous rencontrerons différentes collocations que l'apprenant doit connaitre et savoir utiliser à bon escient : au bon moment (la bonne situation de communication) et au bon endroit (l'emploi linguistique correct) :

la joie de réussir un examen : être aux anges, sauter de joie...

la joie de fêter un anniversaire : faire plaisir, être content...

\footnotetext{
${ }^{4}$ Notons que pour ces deux derniers registres, les exemples pourraient être inversés étant donné que les lexies « gueule » et »troncher » sontdavantage vulgaires que « fendre » et « poire ».
} 
Ces exemples nous permettrons d'avancer vers un enseignement onomasiologique du lexique. Les tentatives d'enseignement sémasiologique des expressions n'ont pas été concluantes ; en effet, donner aux apprenants des listes d'expressions - présentées sous formes d'exercices variés - contenant un nom ou un verbe d'affect (toutes les expressions contenant le verbe « pleurer» ou le substantif « cœur »), n'aide que faiblement à la mémorisation de leur sens. La forme peut-être retenue car répétée, cependant on rencontre souvent des apprenants qui se souviennent d'une expression contenant un verbe en particulier pour l'avoir abordé dans une liste sans savoir du tout dans quel contexte l'utiliser. Un abord onomasiologique permet d'aborder non pas les expressions contenant le mot "pleurer» mais celles signifiant " pleurer » (ou « être triste » de façon plus générale) sans forcément contenir le verbe d'affect (avoir le cafard ne contient pas de lexies des affects tout en y étant lié sémantiquement).

Pour le FLE, nous privilégions l'enseignement de l'expression de tel ou tel affect dans telle situation de communication. Ce n'est pas seulement la place de la phraséologie des affects dans les discours qui est en jeu, mais la façon dont les discours appellent telle ou telle phraséologie pour exprimer les affects.

\subsection{Les propositions didactiques existantes}

Les manuels de FLE ne proposent que de rares expressions figées culturellement marquées et donc souvent présentées de façon anecdotique dans une rubrique un peu à part et seulement dans des niveaux avancés de l'apprentissage de la langue. Notons que les manuels récemment parus contiennent désormais davantage d'expressions figées, voire un chapitre sur l'expression des affects, ce que proposent Merieux et al. (2005: 40, 118). Il existe un seul ouvrage consacré à l'enseignement des expressions figées, celui de Galisson (1984). L'ouvrage est un précieux recueil d'exercices imaginatifs pour tenter de faire deviner les nombreuses expressions présentées aux apprenants (utilisation de dessins, de rébus, de dictionnaire spécialisé etc.). Les exercices proposés conviennent à des apprenants d'un niveau avancé en français (B2 à $\mathrm{C}$ ) et font appel aux représentations iconiques des expressions, ce qui est fort intéressant pour aider à fixer le sens grâce à la mémoire visuelle : par exemple, pour l'expression Donner un coup d'épée dans l'eau, il y a le dessin d'un homme frappant de l'eau dans une bassine à l'aide d'une épée. L'ouvrage est à utiliser avec un dictionnaire d'expressions figées (Galisson, 1991) qui aide à la compréhension et à l'utilisation; sans ce dictionnaire, les exercices hors contexte deviennent très difficiles à manipuler. Ce dictionnaire est à la fois sémasiologique et onomasiologique, ce qui permet de retrouver les expressions par le mot ou par la notion. Ceci a un intérêt didactique non négligeable absent des dictionnaires courants d'expressions figées (mais là n'est pas leur fonction). Nous aurions toutefois deux réserves à propos de l'ouvrage d'exercices (dont nous nous servons régulièrement): 1/ l'incohérence dans la présentation des expressions, parfois un verbe, d'autres fois pas, ce qui a tendance à dérouter les apprenants et à les conduire vers de fausses hypothèses morphosyntaxiques souvent inutiles ; 2/ les expressions figées sont présentées par les thèmes des éléments composant l'expression (celles qui contiennent le lexique des animaux, celui des végétaux, des personnages historiques...), et non à partir de la notion exprimée par l'expression complète, ce qui nuit à leur utilisation. Les apprenants sont davantage tentés de connaître les expressions pour dire sa joie plutôt que celles qui contiennent le mot ange sans toujours bien comprendre dans quelle situation de communication les utiliser. Nous verrons ci-dessous les solutions envisagées pour palier ce défaut. 
Quant aux collocations, on en trouve tout au long des consignes, des textes, des exercices des manuels, mais elles ne font que très rarement l'objet d'un enseignement spécifique. Il paraîtrait important de s'arrêter sur certaines d'entre elles pour préciser notamment que leur forme et leur morphosyntaxe peut être variable; voici des exemples trouvés dans des manuels :

Un texte de compréhension dans Rond-point (Labascoule et al., 2004 : 78), qui contient les collocations suivantes sans les traiter pour les enseigner : organiser une compétition, une opération publicitaire.

Une consigne dans Vocabulaire progressif du français (Schmitt, $2004: 12)$ : remettre en ordre.

Nous pourrions penser que la fréquente apparition de certaines de ces collocations dans les consignes des manuels facilite leur mémorisation. Cependant, on constate que les apprenants ne retiennent pas seuls ces structures et ont besoin d'une mise en exergue de la phraséologie pour la retenir. A quels moments doit-on alors les aborder? Qu'en est-il des affects et de la phraséologie associée?

Dans son ouvrage, Lewis (2000) propose d'enseigner les collocations à l'aide de corpus électroniques. De nombreux auteurs abondent dans ce sens, comme Binon et al. (2003) et Verlinde et al. (2006). Les contextes et les concordanciers ${ }^{5}$ sont à l'honneur. Ces outils permettent de visualiser rapidement les occurrences et les constructions d'une association lexicale particulière. Ainsi apparaissent toutes les constructions d'un élément linguistique, ce qui est fort pertinent pour les collocations associées à une lexie particulière. Prenons l'exemple des occurrences du nom joie pour avoir la liste de ses collocations ${ }^{6}$ :

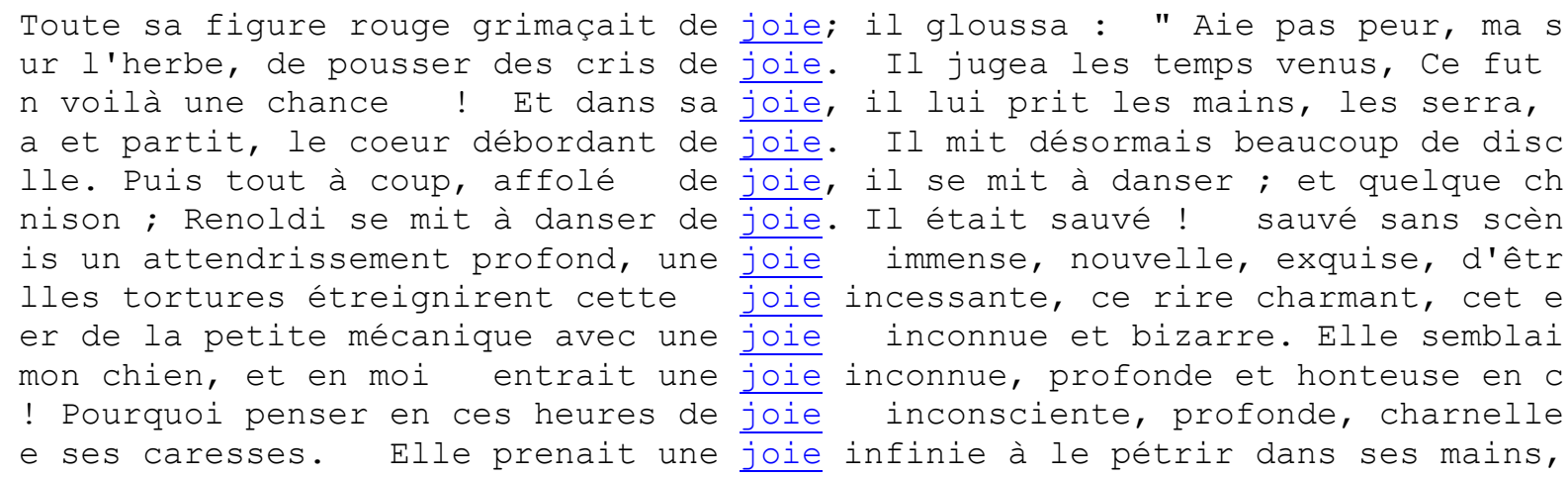

Ces listes de d'exemples sont à manipuler avec précaution dans l'enseignement, en fonction du niveau en langue des apprenants. L'exemple précédent permet de lister les verbes associés à un nom d'affect particulier, de donner les variations morphosyntaxiques possibles, mais n'aide que partiellement à extraire le sens de la collocation ; ce qui sera moins difficile pour des apprenants de niveau avancé. Ceci peut être dommageable dans l'enseignement en raison

5 Concordancier ou «table de concordances » : listes d'exemples pris dans des contextes différents à l'aide de grands corpus de textes informatisés.

${ }^{6}$ Exemples pris dans un concordancier gratuit en ligne, The Compleat Lexical Tutor, extraits de Maupassant: http://www.lextutor.ca/concordancers/concord_f.html 
de l'opacité sémantique de certaines collocations (cf. peur bleue). Enfin, les concordances permettent l'extraction d'une association lexicale mais pas d'un sens. Il n'est pas possible de rechercher les structures qui signifient la joie sans contenir le mot joie; de ce fait, nous passerions à côté de collocations du type être aux anges ou être au $7^{e}$ ciel. C'est pour cette raison qu'il est difficile d'extraire des expressions figées associées aux noms affects car ces derniers n'y apparaissent pas : le nom peur n'est pas dans prendre ses jambes à son cou.

Ces deux approches, l'une à l'aide de corpus et l'autre de dictionnaires, se complètent puisque la première privilégie la forme tandis que l'autre s'attache aussi au sens. Tous les aspects peuvent ainsi être développés par l'enseignant: les formes syntaxiques (le corpus et le dictionnaire), les variations morphologiques (le corpus) et le sens (le dictionnaire), ce qui permet d'envisager une variété importante d'exercices. Sur ce plan, la Base lexicale du français $^{7}$ développée par Binon et Verlinde est une parfaite adéquation entre corpus et dictionnaire puisque les deux outils sont largement exploités et permettent la présentation très détaillée de nombreuses collocations. Reste toutefois à trouver les collocations et les expressions figées qui ne contiennent pas de noms d'affects tout en signifiant un affect.

\subsection{Quelques propositions didactiques}

Partant de la question «comment trouver toutes les expressions figées et les collocations des affects qui ne contiennent pas de noms ou de mots d'affects? », et pour résumer : comment exprime-t-on les affects en français ?, nous avons alors envisagé non pas d'enseigner le lexique des affects, mais le lexique qui permet d'exprimer les affects. Cette perspective onomasiologique de l'enseignement du lexique ouvre un accès par les sens, par exemple donner toutes les collocations qui expriment la tristesse ou la colère comme avoir le cour gros, avoir le cafard, pleurer toutes les larmes de son corps, en avoir ras le bol, sortir de ses gonds...

\section{a. Des images pour les expressions}

L'enquête menée par Labre à propos de l'opacité sémantique a conduit l'auteur à élaborer une séquence didactique autour de quelques expressions figées et collocations. Elle a utilisé l'outil TICE et en particulier, le logiciel HotPotatoes. Les exercices sont de type à trou, QCM ou d'appariement et Labre a choisi de les compléter soit à l'aide d'images illustrant l'expression ou la collocation, soit à l'aide de petites définitions adaptées pour des apprenants de niveau B1-B2 en langue.

«Ces images ont pour but de proposer une représentation iconique du sens des mots afin que la mémorisation en soit plus efficace remédiant ainsi au manque de connaissance lexicale et étymologique des apprenants. L'image ne suffisant pas et pouvant être un élément cognitif obsolète pour certains apprenants, un commentaire étymologique explicite vient renforcer la compréhension de ces expressions figées et collocations. » (Labre, $2006: 63$ )

Voici l'exemple d'un des exercices proposés par Labre, où la bonne réponse contient du texte et de l'iconique (quand cela est possible, une image illustre l'expression) afin d'aider au mieux les apprenants à cerner le sens de l'expression :

Les expressions/collocations et leur signification. Questions à choix multiples.

\footnotetext{
${ }^{7}$ http://ilt.kuleuven.be/blf/\#
} 
Vous venez de voir les différents sentiments et les avez associés à des images. Conservez- les dans un coin de votre tête. Maintenant, vous allez en savoir un peu plus sur les expressions et collocations que nous avons vues en classe.

Cliquez sur la ou les bonnes réponses, un commentaire s'affichera ; lisez-le et vous en saurez plus...

1. pleurer comme une fontaine signifie

1. pleurer de l'eau

2. pleurer beaucoup

3. pleurer bruyamment

2. pleurer comme une Madeleine signifie

1. pleurer beaucoup

2. pleurer en mangeant un gâteau

3. pleurer comme ma voisine qui s'appelle Madeleine

3. En avoir gros sur la patate signifie

1. manger une pomme de terre énorme

2. être triste

3. être très triste...

Le choix de la phraséologie s'est fait à l'aide de plusieurs dictionnaires d'expressions figées et de collocations. Labre a toutefois choisi d'intégrer à sa séquence des expressions et des collocations de tous les registres de langue; ainsi, trouve-t-on avoir la trouille ou avoir les foies à côté de avoir peur. La séquence ${ }^{8}$ compte sept exercices (4 de compréhension et 3 de production) et vise le développement de stratégies de déduction du sens des expressions figées et surtout des collocations. Des expressions et collocations synonymes ou proches notionnellement sont présentées afin d'apprendre à nuancer l'expression de ses affects selon les situations de communication et surtout, selon les registres de langue : avoir le béguin pour quelqu'un, avoir un ticket avec quelqu'un, faire une touche.

L'appariement répété de ces structures tend à forcer l'esprit à retenir les combinaisons lexicales complètes. Ceci aide, si ce n'est à la mémorisation de toutes les expressions et collocations proposées, à comprendre qu'il existe un lexique un peu particulier composé de métaphores (les images sont d'un grand secours pour cet aspect-là), avec des structures syntaxiques particulières et dont l'usage est fréquent par les LN. Cette prise de conscience de la part des LNN leur permet de ne plus envisager la compréhension et leur production langagière en "mots isolés", mais en associant des lexies selon des règles lexicales, syntaxiques et sémantiques.

Tout LN, de quelque langue que ce soit, possède son propre répertoire culturel. Les LNN ont moins facilement accès à ce savoir culturel qui doit être appris. Nous avons noté (grâce à la définition donnée par les personnes interrogées) qu'une certaine imperméabilité d'accès à la référence culturelle, présente dans les expressions figées et collocations, freine leur utilisation.

\section{b. Des mises en contextes pour des collocations}

Nous avons imaginé un enseignement par entrées notionnelles pour six affects : la joie, la peur, la tristesse, la colère, honte et la jalousie (Cavalla et al., 2005). Le choix de ces affects suit les critères de classements en psychologie: sentiments/émotions primaires, mixtes...(Colletta et al., 2003, Rime et al., 1989). C'est en rassemblant plusieurs types de documents tels que le thésaurus de Péchoin (Péchoin, 1992), le Petit Robert (Rey, 1994b) et

8 Elle est en ligne à l'adresse suivante : http://opus.grenet.fr/dokeos/cuef/index.php puis «Expressions idiomatiques et FLE / séquences ». 
des ouvrages didactiques (notamment celui de Galisson sur les expressions imagées) que nous avons établi une liste - non exhaustive - de la phraséologie de ces affects. Le niveau en langue " intermédiaire » des apprenants visés a contraint à la non exhaustivité et à certains choix lexicaux. Nous avons tenté d'extraire les collocations et les expressions figées qui nous semblaient être les plus courantes ; au final, il apparaît que nous avons sélectionné davantage de collocations que d'expressions figées. Ceci reflète une certaine réalité langagière que nous n'avons qu'évoquée et qui semble évidente. Les exercices imaginés entrent dans des thèmes parfois très différents. En effet, comme dit précédemment, on n'exprime pas ses sentiments et ses émotions de la même façon selon la situation de communication. Nous avons alors envisagé plusieurs situations pour un même affect afin que les collocations et les expressions figées rencontrées soient enseignées dans les contextes adéquats. Par exemple, dans le chapitre de la peur sont abordés tour à tour, les films d'horreur, les phobies, les ambiances qui font peur (Cavalla, et al., 2005) :

\section{Exercice 46 Paul et les peurs : Lisez les informations et retrouvez les peurs des amies de Paul.}

Aujourd'hui, Paul a rendez-vous avec ses amies Léa, Myriam, Clara, Elsa et Marion. Il doit aller dans cinq lieux différents de Grenoble, à cinq heures différentes et il veut faire peur à chacune de ses amies. Mais, il a perdu son agenda et il ne sait plus qui il doit rencontrer, à quelle heure et où. De même, il ne sait plus quelles sont les peurs de chacune de ses amies.

Heure des rendez-vous : 10 heures, 14 heures, 18 heures, 20 heures, 22 heures.

Noms des lieux : le musée d'Histoire Naturelle, la Place Grenette, l'église Saint Laurent, les bulles de Grenoble (le téléphérique), le Jardin de Ville.

En fin d'après midi, dans le jardin fleuri, Clara est debout sur un banc en train de chasser Max, le gros chien de Paul qui est content de la voir.

Myriam adore les animaux mais il ne faut pas lui parler de moustiques ! Elle est libre ce week-end à l'heure de la sieste. Elle adore l'art.

Léa travaille très tard même si elle déteste sortir la nuit. Les monuments religieux la passionnent.

Paul donne rendez-vous à Elsa à 20 heures devant la fontaine, sur la place, pour aller au restaurant. Le jour de la fête de la musique tout le monde est de sortie : elle tremble de peur.

L'une d'entre elles a le vertige, pourtant Paul veut l'emmener voir les Alpes du haut de la Bastille en téléphérique.

\begin{tabular}{|l|l|l|l|}
\hline & Où ? & A quelle heure ? & Quelle peur ? \\
\hline Léa & & & \\
\hline Myriam & & & \\
\hline Clara & & & \\
\hline Elsa & & & \\
\hline Marion & & & \\
\hline
\end{tabular}

\section{Exercice 47 Les situations de peur.}

Imaginez chaque situation où Paul se retrouve avec l'une de ses amies qui a peur. Rédigez le dialogue entre Paul et son amie en utilisant les expressions de la peur.

Le réinvestissement de la phraséologie est systématique dans l'ouvrage dès l'instant où comme nous l'avons vu - il est important de mettre en contexte ces éléments pour des raisons morphologiques et syntaxiques notamment. C'est alors que les apprenants découvrent au fil des exercices qu'ils doivent retenir l'association lexicale entière et pas seulement le nom du sentiment. Les textes donnés leur montrent que les noms d'affects laissent souvent la place à des expressions et des collocations, des ambiances (un château hanté et des fantômes pour la peur par exemple) et des réactions physiques ou des comportements (rougir pour la honte ou 
crier pour la colère). Nous pourrions d'ailleurs approfondir cet aspect en utilisant les descriptions de Tutin : « les manifestations invalidantes (trembler, suffoquer, défaillir,...) sont particulièrement fréquentes avec des affects intenses et négatifs" (Tutin, et al., $2006: 42$ ). En ajoutant les structures associées, les apprenants auront les patrons syntaxiques et sémantiques de ces collocations ; ainsi pourront-ils, peut-être, acquérir davantage un savoir-faire lexical (manipuler les structures et les sens à bon escient) qu'un savoir lexical isolé souvent handicapant pour le réemploi.

Enfin, cet ouvrage nous a permis de voir que, dans notre société, les affects s'expriment aussi à l'aide du corps et de la voix. Nous avons donc proposé quelques rares exercices faisant appel à la gestuelle associée et à l'intonation spécifique à certains affects. Les apprenants étrangers ne perçoivent pas toujours bien ces aspects culturels qu'ils ont alors tendance à éviter de peur de ne pas « savoir-être » dans la culture qu'ils découvrent.

\section{c. Des images et des contextes}

Dans le cadre d'un projet ESC (Ecole et Sciences Cognitives ${ }^{9}$ ), nous avions conclu une partie du projet avec des séquences didactiques pour des enfants LN de 9-11ans. L'objectif était de leur enseigner le lexique des affects en vue d'une aide à la rédaction (Boch et al., 2005, Grossmann et al., 2003). Après une expérimentation sur plusieurs heures dans des classes, nous avons élaboré un site ${ }^{10}$ qui résume les besoins qui nous sont apparus importants pour les enfants LN de cet âge. La séquence proposée commence par la découverte des noms de sentiments et chemine entre les expressions figées, les collocations, les manifestations physiques, les éléments d'ambiance, quelques structures grammaticales pour aboutir à une production écrite des enfants. L'objectif didactique et linguistique est davantage dans la variation lexicale que dans la "meilleure façon de l'écrire »; à la suite de Grésillon (dans Authier-Revuz et al., 2004 : 12), nous considérons que varier le lexique permet aussi de « faire mieux » dès l'instant où si le scripteur a deux fois plus de lexies à sa disposition, il pourra multiplier d'autant ses possibilités d'écriture. En d'autres termes, il aura acquis une meilleure capacité d'écriture et de choix lexicaux pour la réécriture. Il s'est avéré que cette variété et ces objectifs ont séduit des enseignants de FLE, LNN, qui l'ont jugé abordable par des apprenants d'un niveau intermédiaire.

La séquence, qui associe images et mises en contexte dans des contes qui font peur aux enfants, adopte une démarche onomasiologique dans des situations où les affects (et notamment la peur sur laquelle nous avions mis l'accent) sont très présents chez les personnages. Cette séquence a révélé qu'il est autant difficile pour un LN que pour un LNN d'exprimer les sentiments de personnages fictifs, aussi bien à l'oral qu'à l'écrit. L'exercice final de rédaction d'une histoire qui fait peur a été repris à l'identique dans l'ouvrage Emotions-Sentiments pour les étudiants étrangers. On retrouve étrangement ches les LNN les mêmes défauts d'écriture chez eux que chez les enfants : peu de sentiments décrits et exprimés, beaucoup d'ambiance spécifique au sentiment, beaucoup de manifestations physiques.

\footnotetext{
${ }^{9}$ «Favoriser le développement des compétences lexicales et métalexicales en vue d'une aide à la production de textes au cycle 3 et au collège (2003 -2006) », projet financé par le Ministère de la Recherche ; dirigé par F.Grossmann au Lidilem en collaboration avec les laboratoires Labécd (EA 3259) de Nantes, PsyCLE (EA 3273) d'Aix en Provence et l'OLST de Montréal.

${ }^{10} \mathrm{http}: / / \mathrm{w} 3 . \mathrm{u}$-grenoble3.fr/lidilem/projets/ESC/
} 
La rédaction de cette histoire par les étudiants étrangers est une sorte d'aboutissement. Aussi y avons-nous ajouté une contrainte qui les oblige à envisager l'expression des affects dans son ensemble : ils doivent la jouer. L'oralisation leur donne l'occasion d'exprimer les affects linguistiquement, avec une intonation particulière et une gestuelle appropriée. De ce fait, les affects sont éprouvés par les apprenants étrangers qui en profitent pour créer des expressions ou des collocations souvent très imagées.

\section{Discussion}

L'approche didactique omasiologique que nous privilégions permet de présenter les associations lexicales en contextes variés et par notion. Pour dresser des listes des expressions et des collocations des affects, tous les classements, les recherches et les ouvrages didactiques évoqués nous ont servi et nous serviront encore. Ces deux problématiques de la description linguistique de la phraséologie et de son enseignement s'associent depuis plusieurs années à l'acquisition des langues secondes en milieu scolaire (Germain, 2001). Nous sommes alors tentées d'aller glaner des informations dans ce domaine vaste qu'est l'acquisition mais qui pourrait peut-être nous fournir de nouvelles pistes pour un enseignement/apprentissage davantage optimisé pour ces phénomènes linguistiques si particuliers.

Nous serions alors d'avis de donner aux apprenants les outils qui leur permettraient de construire eux-mêmes leurs compétences lexicales. Le lexique n'est pas une "liste » de lexies, mais un ensemble structuré d'éléments contraints par des règles strictes et très variables d'une langue à l'autre. Si l'enseignant donnait la possibilité à l'apprenant d'entrer dans ces structures spécifiques du lexique et d'apprendre à les manipuler, alors, ce dernier serait à même de développer ses propres compétences lexicales. Une telle approche consiste à donner aux apprenants, en plus d'un savoir lexical, une compétence qui leur permettra de prendre en main leur savoir afin qu'ils puissent manipuler la langue à leur guise (Petiot et al., 2005). Un tel enseignement tend à développer des savoir-faire plus que des savoirs isolés : dès l'instant où l'apprenant saisit les règles, alors il peut les manipuler dans toutes les situations de communication. Nous rencontrons cette approche lexicale dans des méthodes d'anglais langue étrangère (Chini, 2005) où les apprenants (des collégiens francophones dans ce cas) sont invités à apprendre certaines règles morphologiques de l'anglais afin de manipuler seuls les éléments constructifs de la langue. Nous commençons à comprendre comment construire un enseignement onomasiologique pour les expressions associées aux affects et nous souhaiterions associer cet enseignement à celui de savoir-faire linguistiques.

\section{Références}

Authier-Revuz, J., Gresillon, A. (2004). La réécriture. Questions théoriques. Le Français aujourd'hui, $\mathrm{n}^{\circ}$ 144. pp. 9-17.

BASSANO, D. (2005). Le développement lexical précoce : état des questions et recherches récentes sur le français. In Grossmann, F., Paveau, M.-A. \& Petit, G. (dir.). Didactique du lexique, cognition, discours. Grenoble: Ellug.

BIDAUD, F. (2006). Les titres de presse médiateurs pour l'enseignement de la langue et la culture françaises. Dialogues et cultures, $\mathrm{n}^{\circ}$ 51. pp. 81-85.

BINON, J. (2000). Un quart de siècle d'enseignement / apprentissage de français des affaires 1

- Du dogmatisme au pluralisme didactique. Romaneske, en ligne: http://www.vlrom.be/index.php?option=com content\&task=view\&id=184\&Itemid=48 
BinOn, J., VerLinde, S. (2003). Les collocations : clef de voûte de l'enseignement et de l'apprentissage du vocabulaire d'une langue étrangère ou seconde. La lettre de l'AIRDF, $\mathrm{n}^{\circ} 33$. pp. 31-36.

Boch, F., Cavalla, C. (2005). Evaluer l'expression des sentiments dans des textes d'enfants, une mission impossible?. Repères, $\mathrm{n}^{\circ} 31$. pp. 55-71.

Cavalla, C., CROZIER, E. (2005). Emotions-Sentiments. Grenoble: Presses Universitaires de Grenoble.

Cavalla, C., Crozier, E. (2005). Expérience d'enseignement de l'expression des émotionssentiments en classe multiculturelle de FLE. Diversités culturelles et apprentissage du français, ss.dir. Bertrand, O. Paris: Editions Ecole Polytechnique.

Cavalla, C., Grossmann, F. (2005). "Caractéristiques sémantiques de quelques 'Noms scientifiques' dans l'article de recherche en français". Akademisk prosa, ss.dir. Kin, T. Bergen: Skrifter fra KIAP Romansk institutt, Universitetet i Bergen.

CHINI, D. (2005). Entre savoirs déclaratifs et stratégies procédurales : le lexique dans l'enseignement-apprentissage de l'anglais. Didactique du lexique, cognition, discours, ss.dir. Grossmann, F., Paveau, M.-A., Petit, G. Grenoble: Ellug.

Colletta, J.-M. \& TCherkassof, A. (2003). Les émotions : cognition, langage et développement. Sprimont: Mardaga.

CowIE, A.P. (1978). The place of illustrative material and collocations in the design of a learner's dictionary. Oxford: Oxford University Press.

GALISSON, R. (1984). Les expressions imagées. Paris: Clé International.

GALISSON, R. (1991). Dictionnaire de compréhension et de production des expressions imagées. Paris: Clé International.

GASS, S.M., SELINKER, L. (2001). Second language acquisition : an introductory course. New Jersey: Mahwah, Lawrence Erlbaum.

GERMAIN, C. (2001). La didactique des langues : une autonomie en devenir. Recherches et Applications, $\mathrm{n}^{\circ}$ spécial. pp. 13-23.

GonZALEZ-REY, I. (2002). La phraséologie du français. Toulouse: Presses Universitaires du Mirail.

Grossmann, F., Boch, F. (2003). Production de textes et apprentissage lexical : l'exemple du lexique de l'émotion et des sentiments. Repères, ${ }^{\circ}$ 28. pp. 117-135.

Grossmann, F., Tutin, A. (2003). Les collocations : analyse et traitement. Amsterdam: De Werelt.

HAUSMANN, F.J. (1989). Le dictionnaire de collocations. Wörterbücher : ein internationales Handbuch zur Lexicographie. Dictionaries. Dictionnaires, ss.dir. Hausmann F.J., Reichmann, O., Wiegand, H.E., Zgusta, L. Berlin/New-York: De Gruyter.

LabAscoule, J., LAuSe, C., RoYer, C. (2004). Rond-Point 1 - Méthode de français basée sur l'apprentissage par les tâches. Grenoble, Barcelone: Presses Universitaires de Grenoble Difusión.

LABRE, V. (2006). Expressions figées et collocations des sentiments : analyse didactique pour la classe de FLE. Mémoire 1re année Master Français Langue Etrangère Professionnel, ss.dir. Cavalla, C. Université Stendhal-Grenoble3.

LEWIS, M. (2000). Teaching collocation : Further developments in the lexical approach. Hove: Language teaching publications LTP.

MEL'CÛK, I., WANNER, L. (1996). Lexical Functions and Lexical Inheritance for Emotion Lexemes in German. Lexical Functions in Lexicography and Natural Language Processing, ss.dir. Wanner, L. Amsterdam/Philadelphia: ed. John Benjamin.

MerieuX, R., Loiseau, Y., Bouvier, B. (2005). Connexions 3. Paris: Didier.

Pecholn, D. (1992). Thésaurus : Des idées aux mots, des mots aux idées. Paris: Larousse. 
Petiot, G., Reboul-Toure, S. (2005). Apprentissage et enseignement du lexique : pour une didactique mettant en oeuvre les discours et la langue. Didactique du lexique, cognition, discours, s.dir. Grossmann, F., Paveau, M.-A., Petit, G. Grenoble: Ellug.

REY, A. (1994). Nouveau Petit Robert, Dictionnaire alphabétique et analogique de la langue française. Paris: Dictionnaires le Robert.

RIME, B., SCHERER, K. (1989). Les émotions. Neuchâtel: Delachaux et Niestlé.

Schmitt, S. (2004). Vocabulaire progressif du français - Pour adolescents - Niveau débutant. Paris: Clé International.

SIEPMAN, D. (2006). Collocations et dictionnaires d'apprentissage onomasiologiques bilingues : questions aux théoriciens et pistes pour l'avenir. Langue Française, ${ }^{\circ} 150$. pp. 99-117.

Tutin, A. (2007). Collocations du lexique transdisciplinaire des écrits scientifiques : annotation et extraction des propriétés linguistiques dans la perspective d'une application didactique. Cahiers de l'Institut de linguistique de Louvain, $\mathrm{n}^{\circ}$ Vol. 31, $\mathrm{n}^{\circ}$ 2-4. pp. 247-262.

Tutin, A., Grossmann, F. (2002). Collocations régulières et irrégulières : esquisse de typologie du phénomène collocatif. Revue Française de Linguistique Appliquée, $\mathrm{n}^{\circ}$ VII. pp. 7 25.

Tutin, A., Novakova, I., Grossmann, F. \& Cavalla, C. (2006). "Esquisse de typologie des noms d'affect à partir de leurs propriétés combinatoires". Langue Française, n ${ }^{\circ} 150$. pp. 3249.

Verlinde, S., Binon, J., Selva, T. (2006). Corpus, collocations et dictionnaires d'apprentissage. Langue Française, ${ }^{\circ}$ 150. pp. 84-98. 